\title{
ASSOCIATIONS BETWEEN SLEEP DISTURBANCES, PERSONALITY, AND TRAIT EMOTIONAL INTELLIGENCE
}

\author{
by \\ SARAH ELIZABETH EMERT \\ KENNETH L. LICHSTEIN, COMMITTEE CHAIR \\ BEVERLY E. THORN \\ ADAM P. KNOWLDEN
}

\begin{abstract}
A THESIS
Submitted in partial fulfillment of the requirements for the degree of Master of Arts in the Department of Psychology in the Graduate School of The University of Alabama
\end{abstract}

TUSCALOOSA, ALABAMA 
Copyright Sarah Elizabeth Emert 2016 ALL RIGHTS RESERVED 


\begin{abstract}
Insomnia is defined by difficulty falling asleep, staying asleep, or waking earlier than desired with inability to return to sleep. Complaints of nonrestorative sleep (NRS) are often associated with insomnia but can occur independently. Fragmented sleep and NRS have been shown to relate to one's mood, one's ability to process their own or others' emotions, and can lead to changes in cognitions and behaviors. Personality traits related to increases in anxiousness may play a role in the development and maintenance of sleep disorders and associated daytime impairment of NRS. The relations between sleep disturbance, personality traits, and trait emotional intelligence are underrepresented in the current literature and findings have been mixed. This study addressed some inconsistencies by identifying associations between the Big Five personality traits, trait emotional intelligence (TEI), complaints of NRS, and disrupted sleep associated with insomnia. We predicted that neuroticism would relate to poorer sleep and that conscientiousness and TEI would be associated with better sleep. Openness to experience, extraversion, and agreeableness are not often discussed in the literature, but were expected to associate similarly as conscientiousness. Results provided support for the idea that trait characteristics are associated with insomnia severity and restorative sleep quality. These findings indicated that personality and TEI may play a role in development and maintenance of sleep disorders and daytime impairment of NRS; higher conscientiousness, lower neuroticism, and higher TEI possibly demonstrate a protective role to experiencing negative effects of poor sleep.
\end{abstract}




\section{LIST OF ABBREVIATIONS AND SYMBOLS}

$\alpha \quad$ Cronbach's index of internal consistency

$\beta \quad$ Beta coefficient: standardized regression coefficient

$B \quad$ Unstandardized regression coefficient

SE Standard error of the estimate: measure of the accuracy of predictions made by a regression line

$F \quad$ Fisher's $F$ ratio: a ratio of two variances

$n \quad$ Sample size

$M \quad$ Mean: the sum of a set of measurements divided by the number of measurements in the set

SD Standard deviation: measure of the variation of a set of data values from its mean

$p \quad$ Probability associated with the occurrence of obtaining a value equal to or greater than the observed value under conditions of the null hypothesis

$r \quad$ Pearson product-moment correlation coefficient: measure of the linear correlation between two variables

$R^{2} \quad$ Coefficient of determination: proportion of total variation explained by a regression model

$\Delta R^{2} \quad$ Incremental increase of $R^{2}$ from one step of a regression model to another 


\section{ACKNOWLEDGEMENTS}

First, I wish to thank my mentor and thesis committee chair, Kenneth Lichstein, for his encouragement and guidance throughout the course of this project. His mentorship and expertise, in combination with the work involved in the composition of this thesis, has allowed for an incredibly rewarding experience. I would also like to express thanks to my other committee members, Beverly Thorn and Adam Knowlden, for their interest, support, and feedback on this project. Additionally, I would like to show gratitude to all of the psychology student volunteers at The University of Alabama without whom I could not have completed this project. Finally, I wish to thank my family, especially my parents, whose love and support I am inexpressibly grateful for. Without their reassurance and encouragement I would not be where I am today. 


\section{CONTENTS}

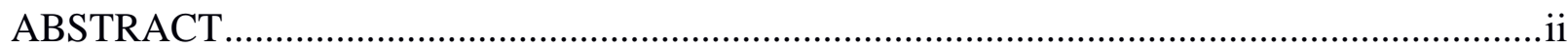

LIST OF ABBREVIATIONS OR SYMBOLS ……...........................................................ii

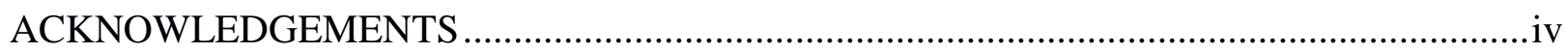

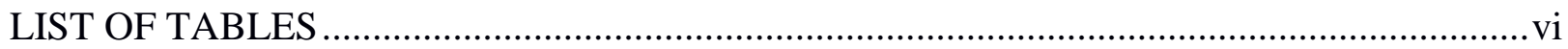

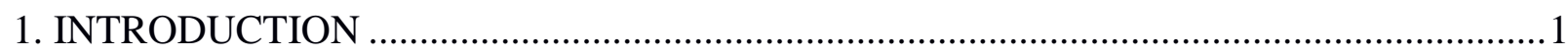

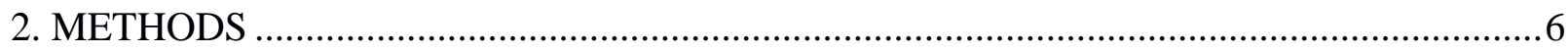

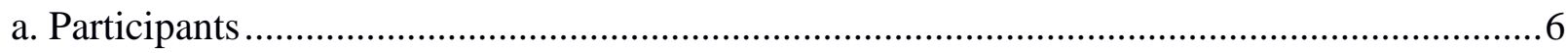

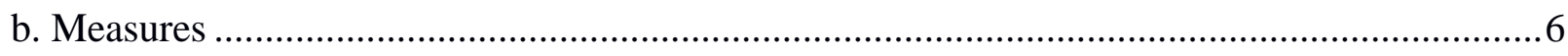

c. Procedure

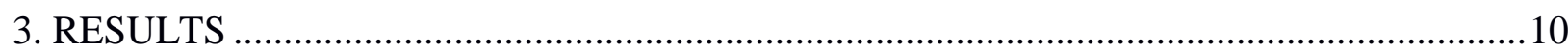

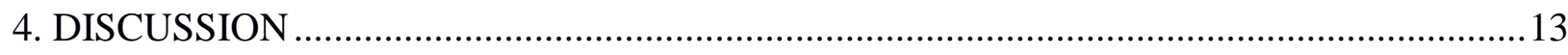

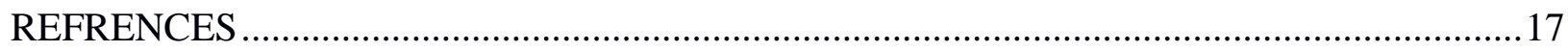

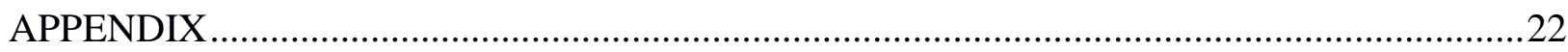

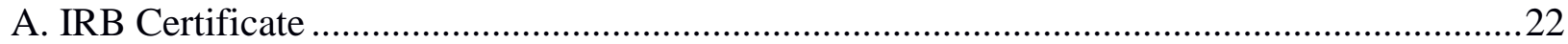




\section{LIST OF TABLES}

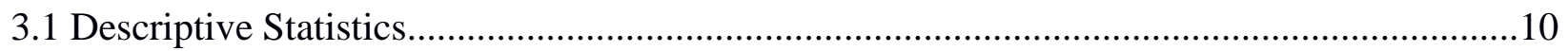

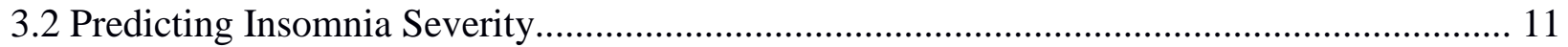

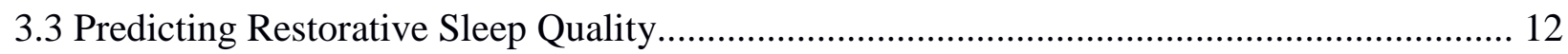




\section{CHAPTER 1: INTRODUCTION}

Insomnia disorder is a prevalent sleep disorder, occurring in almost $16 \%$ of the population, characterized by: a continuously occurring complaint of difficulty initiating sleep, maintaining sleep, or early morning awakening with the inability to return to sleep; related daytime impairment; and concerns or dissatisfaction with sleep (American Academy of Sleep Medicine [AASM], 2014; American Psychiatric Association [APA], 2013; Lichstein, Durrence, Riedel, Taylor, \& Bush, 2004). Nonrestorative sleep (NRS), a complaint of poor sleep quality that does not leave an individual rested upon awakening despite adequate duration, is often associated with a diagnosis of insomnia disorder (APA, 2013). However, complaints of NRS have also been identified without other symptoms of insomnia, occurring with normal sleep initiation, maintenance, and duration (Ohayon, 2005; Ohayon \& Roth, 2001; Roth et al., 2006, 2010). The role of NRS in diagnosing insomnia has varied over the history of diagnostic systems. At present, it remains a component of insomnia disorder within the DSM-5 but was removed in the development of the ICSD-3 (AASM, 2014; APA, 2013). This discrepancy signifies the importance of research aiming to identify factors specific to insomnia and complaints of NRS.

Personality traits are one factor of interest. Findings within the literature related to associations between personality differences and sleep disruption are mixed; however, there seems to be a general trend that those with sleep disturbances or poor sleep quality display more traits of neuroticism, internalization, anxiety, and traits associated with perfectionism (Harvey, Gehrman, \& Espie, 2014; Ohayon, 2005; van de Laar, Verbeek, Pevernagie, Aldenkamp, \& 
Overeem, 2010; Williams \& Moroz, 2009). Individuals high in traits related to neuroticism may be particularly vulnerable to stress-related sleep disruption which could play a causal role in the development of insomnia (Harvey, et al., 2014; van de Laar et al., 2010; Williams \& Moroz, 2009). These individuals take longer to fall asleep, sleep less deeply, have poorer sleep quality, shorter sleep overall, and have lower rapid eye movement (REM) density compared to those low in these traits (Harvey et al., 2014; Williams \& Moroz, 2009).

Traits of conscientiousness also seem to be associated with sleep disturbance. Conscientiousness is positively related to sleep quality and appears to moderate the extent to which high-neuroticism individuals are vulnerable to the adverse effects of poor sleep, with high-neuroticism/low-conscientiousness individuals experiencing more distress and dysfunction to perceived sleep loss and more worrying and rumination around sleep onset (Harvey et al., 2014; Williams \& Moroz, 2009). Conscientiousness was positively related with better health behaviors overall, possibly indicating that conscientious individuals have healthier sleep related behaviors and therefore less disturbance and complaints about poor sleep quality (Bogg \& Roberts, 2004). Though research identifying possible connections between NRS and personality traits is scarce, research indicates that personality differences exist for those who are formally diagnosed with insomnia and those who subjectively identify as insomniacs. Those classified as “objective insomniacs" displayed high introversion, whereas "subjective insomniacs" showed high neuroticism (Dorsey \& Bootzin, 1997). These findings may extend to NRS, a subjectively identified sleep problem.

Another factor of interest is trait emotional intelligence (TEI). Emotional intelligence, described as the perception of one's ability to identify and regulate one's emotional state, involving abilities such as self-awareness, managing emotions, motivating one-self, empathy, 
and handling relationships, has been conceptualized into two groups: ability emotional intelligence and TEI. The former relates to concrete abilities, the latter includes behavioral dispositions and self-perceived abilities (Goleman, 1995; Petrides \& Furnham, 2001; Salovey \& Mayer, 1990). Evidence shows that TEI is linked to many psychological and behavioral facets, including personality, goal orientation, life satisfaction, affect intensity, alexithymia, and depression (Dawda \& Hart, 2000; Martinez-Pons, 1997; Petrides \& Furnham, 2001). Higher emotional intelligence has been associated with better mental, psychosomatic, and physical health outcomes, with TEI being more strongly associated with mental health than was emotional intelligence ability (Schutte, Malouff, Thorsteinsson, Bhullar, \& Rooke, 2007).

Sleep and emotions are closely linked and their relationship is bidirectional (Kahn, Sheppes, \& Sadeh, 2013). Sleep loss produces temporary changes in cerebral metabolism, cognition, emotion, and behavior consistent with mild prefrontal lobe dysfunction including problems with motivation, empathy, flexibility, planning and organization, behavioral inhibition, emotional blunting and affective dysregulation, and insight (Barrash, Tranel, \& Anderson, 2000; Killgore et al., 2008, Spinella, 2005). Aspects of emotional self-assessment may be adversely affected by sleep deprivation (Killgore et al., 2008). This relationship may extend to additional sleep problems including insomnia and NRS or may constitute a predisposition to sleep difficulties. Sleep loss is related to increases in negative affect and less intense, less frequent positive emotions. A negative emotional state prior to bed may alter one's sleep quality with increases of sleep onset latency, wakefulness after sleep onset, number of awakenings from REM sleep, decreases in total sleep time, sleep efficiency, REM and slow-wave sleep stages (Kahn et al., 2013). Sleep complaints may be more frequent in individuals with low TEI due to an inability to adequately differentiate between symptoms of poor sleep. 
Research identifying characteristics of sleep disturbances and the emotional associations of sleep loss is needed and continues to be understudied (Kahn et al., 2013; Ohayon, 2005; Roth et al., 2010; Vernon, Dugar, Revicki, Treglia, \& Buysse, 2010; Wilkinson \& Shapiro, 2012). Though sleep deprivation has been found to be associated with decreased global emotional intelligence, intra- and interpersonal functioning, stress management, and behavioral coping, the comparison between sleep problems and TEI has not been researched (Killgore et al., 2008). Until recently, a standardized operational definition of NRS had been lacking, with sleep quality often assessed using a single item (Stone, Taylor, McCrae, Kalsekar, \& Lichstein, 2008; Vernon et al., 2010; Wilkinson \& Shapiro, 2012). Drake et al. (2014) developed the patient-reported Restorative Sleep Questionnaire (RSQ) as a reliable and valid measure of NRS complaints permitting research to be conducted with the goal of providing a better understanding of NRS. Finally, there is insufficient data related to the associations between sleep and the other Big Five personality traits (extraversion, agreeableness, openness to experience).

The current study addressed some of these gaps by investigating the associations between the Big Five personality traits, TEI, sleep difficulties associated with insomnia, and NRS quality. It was predicted that the Big Five personality traits and TEI would be associated with sleep problems and complaints of NRS. Specifically predicting that neuroticism would relate to poorer sleep (indicated by greater insomnia severity and worse restorative sleep quality) and that conscientiousness and TEI would be associated with better sleep (indicated by less insomnia severity and better restorative sleep quality). Outcomes may help to address inconsistencies in the literature leading to a better understanding of characteristics related to sleep disruptions in insomnia and complaints of NRS. Such information could aid in improved understanding of the 
associations that one's perceptions of sleep, emotion regulation, and disruptions have on sleep complaints and quality of life. 


\section{CHAPTER 2: METHODS}

\section{Participants}

Undergraduate students were recruited from introductory psychology courses at the University of Alabama and received course credit for participating in this research study. There were no eligibility screening criteria.

\section{Measures}

Health Survey. Participants completed an investigator-designed health survey to obtain demographic information. The form included questions regarding the endorsement of subjective feelings of insomnia, other sleep problems, prescription or over-the-counter sleep medication usage, symptoms related to sleep difficulty, alcohol, tobacco, and caffeine use, usage of other drugs, history of medical or psychiatric disorders, and past/current mental health treatment.

Insomnia Severity Index. Perceptions of insomnia severity were assessed with the Insomnia Severity Index (ISI; Morin, 1993). The ISI is a seven-item self-report questionnaire which measures the severity of one's insomnia problems covering the past two weeks. The measure was scored by adding the responses of all seven items. A score of 0-7 indicates "no clinically significant insomnia"; 8-14 indicates "subthreshold insomnia"; 15-21 indicates "clinical insomnia (moderate severity)"; and 22-28 indicated "clinical insomnia (severe)" (Morin, 1993). The ISI is a valid and reliable self-report measure used to evaluate and quantify perceived sleep disturbance associated with insomnia $(\alpha=0.74)$ with empirical support for the online use of the index to measure self-reported insomnia severity (Thorndike et al., 2011). 
Content validity was supported by a component analysis to capture diagnostic criteria for insomnia (Bastien, Vallières, \& Morin, 2001).

Restorative Sleep Questionnaire. An online Restorative Sleep Questionnaire (RSQ) was used to assess the restorative quality of one's sleep (Drake et al., 2014). The weekly version of the form used (RSQ-W) includes wake time, time completed, and 9 items assessing characteristics of NRS. The RSQ-W total score is calculated as the average of the questionnaire items. Scores range from 0 to 100, with higher scores indicating better restorative sleep. Participants were instructed to complete the RSQ-W referencing the past 14 days, chosen for uniformity with the other measures used.

The RSQ shows good psychometric properties $\left(\alpha>0.90, \mathrm{r}_{\text {test-retest }}>0.80\right)$ and unidimensionality. The RSQ is able to distinguish between healthy controls, patients diagnosed with primary insomnia, and insomnia patients with isolated NRS complaints with normal sleepers reporting sleep that was about one standard deviation more restorative than that of those with NRS complaints (Drake et al., 2014).

The Big Five Inventory. The full length Big Five Inventory (BFI-44) was used to assess the Big Five personality traits (John, Donahue, \& Kentle, 1991; John, Naumann, \& Soto, 2008). The questionnaire contains 44 items designed to measure the Big Five dimensions: extraversion, agreeableness, conscientiousness, neuroticism, and openness to experience. The BFI-44 was compared to two other Big Five measures, the NEO Five-Factor Inventory and the Trait Descriptive Adjectives, demonstrating high equivalence among measures (Rammstedt \& John, 2007). The BFI-44 requires less time and participant burden than the more complex NEO-PI-R (240 items). Additionally, it may be as efficient as and more easily understood than either the 60- 
NEO-Five Factor Inventory or 100-item Trait Descriptive Adjectives (Benet-Martínez \& John, 1998; John et al., 2008; Soto, John, Gosling, \& Potter, 2008).

Scoring of the BFI-44 yields a scaled score for each of the five personality traits and is calculated as the mean of the items included in the calculation for each trait. Scaled scores range from 1 to 5, with higher scores indicating higher levels of the trait.

The Trait Emotional Intelligence Questionnaire. The Trait Emotional Intelligence Questionnaire - Short Form (TEIQue-SF) assessed global TEI (Cooper \& Petrides, 2010; Davey, 2006; Petrides \& Furnham, 2006). The TEIQue-SF is a 30-item self-report measure created based on the longer, 153-item version (Cooper \& Petrides, 2010). The TEIQue-SF was found to have good psychometric properties with most of the items having high discrimination parameters and low threshold parameters, indicating that they are easy to endorse and precise at discriminating individuals across the range of the latent trait (Petrides, 2009; Cooper \& Petrides, 2010).

The TEIQue-SF was scored by averaging total item values. Values range from 1 to 7 , with higher scores indicating higher TEI or better emotion-related traits and self-perceived abilities and dispositions.

\section{Procedure}

Participants enrolled for the study through the psychology subject pool website. They were then presented with an information form, including a description of research, information regarding confidentiality, withdrawal from study, anticipated risks and benefits, and contact information for the principal investigator, advisor, and The University of Alabama Research Compliance officer. If students agreed to participate, they provided their name and email address indicating that they read and understood the study protocol and had an opportunity to ask any 
questions. Participants were then assigned a study ID and provided with a link to the study website. Upon logging in, participants could view and complete study questionnaires. 


\section{CHAPTER 3: RESULTS}

Analyses were conducted in IBM SPSS Statistics versions 20 and 21. Pairwise data were available for all 553 participants. Descriptive statistics are shown in Table 3.1.

Table 3.1

Descriptive Statistics

\begin{tabular}{|c|c|c|c|}
\hline Variable & \multicolumn{2}{|c|}{$n=553$} & $M \pm S D$ \\
\hline Age & 553 & & $18.8 \pm 1.7$ \\
\hline Gender (Male) & 553 & $(129)$ & --- \\
\hline Race & 552 & & --- \\
\hline Caucasian & & 467 & --- \\
\hline African American & & 41 & --- \\
\hline Hispanic/Latino & & 16 & --- \\
\hline Asian & & 14 & --- \\
\hline Mixed/Other & & 14 & --- \\
\hline Sleep medication (Taking) & 553 & $(39)$ & --- \\
\hline TEI & 551 & & $5.06 \pm .74$ \\
\hline Openness to experience & 553 & & $3.35 \pm .60$ \\
\hline Conscientiousness & 553 & & $3.69 \pm .60$ \\
\hline Extraversion & 553 & & $3.27 \pm .81$ \\
\hline Agreeableness & 553 & & $3.95 \pm .60$ \\
\hline Neuroticism & 553 & & $2.85 \pm .78$ \\
\hline ISI & 551 & & $6.80 \pm 4.47$ \\
\hline RSQ & 553 & & $54.68 \pm 18.58$ \\
\hline
\end{tabular}

Hierarchical multiple regression analysis was conducted to test the Big Five personality traits and TEI as predictors of insomnia severity controlling for the presence of sleep medication, gender, and race. Block 1 variables were added using an enter method and was comprised of sleep medication, gender and race; block 2 variables were added using a stepwise method and was comprised of the Big Five personality traits and TEI, resulting in 5 blocks. Dummy coding was used to represent the five levels of race, the Caucasian group was the uncoded group and the other four 
groups were coded 1 . The overall model was significant, $F(10,530)=12.83, p<.001 . R^{2}=.20$, indicating that $20 \%$ of the variance of insomnia severity can be accounted for by this model.

In Table 3.2, the relative strength of the individual predictors in the model are presented. In the final model, five variables were statistically significant. The severity of insomnia related sleep difficulties was related to the use of sleep medication, lower conscientiousness, lower TEI, higher neuroticism, and higher openness.

Table 3.2

Predicting Insomnia Severity

\begin{tabular}{lcccc}
\hline Variables & $\boldsymbol{B}$ & $\boldsymbol{S} \boldsymbol{E}$ & $\boldsymbol{\beta}$ & $\boldsymbol{\Delta R}^{\mathbf{2}}$ \\
\hline $\begin{array}{l}\text { Step 1 } \\
\text { Sleep Medication }\end{array}$ & 3.52 & .73 & $.20 * * *$ & $.044^{* * *}$ \\
$\begin{array}{l}\text { Gender } \\
\text { Step 2 }\end{array}$ & .36 & .45 & .03 & \\
Race \\
Step 3
\end{tabular}

We used the same method to test the Big Five personality traits and TEI as predictors of restorative sleep controlling for presence of sleep medication, gender, and race, resulting in 4 blocks. The overall model was significant, $F(9,531)=24.47, p<.001 . R^{2}=.29$, indicating that $29 \%$ of the variance of restorative sleep quality can be accounted for by this model.

Table 3.3 presents the relative strength of the individual predictors in the model. In the final model, three variables were statistically significant. Nonrestorative sleep is related to higher neuroticism, lower conscientiousness, and lower TEI. 
Table 3.3

Predicting Restorative Sleep Quality

\begin{tabular}{lcccc}
\hline Variables & $\boldsymbol{B}$ & $\boldsymbol{S E}$ & $\boldsymbol{\beta}$ & \multicolumn{1}{c}{$\boldsymbol{\Delta \boldsymbol { R } ^ { \mathbf { 2 } }}$} \\
\hline Step 1 & & & & $.023^{* *}$ \\
Sleep Medication & -7.81 & 3.07 & $-.11^{*}$ & \\
Gender & -4.23 & 1.88 & $-.10^{*}$ & \\
Step 2 & & & & .001 \\
$\begin{array}{l}\text { Race } \\
\text { Step 3 }\end{array}$ & & & & \\
TEI & 11.43 & .98 & $.45^{* * *}$ & $.199^{* * *}$ \\
Step 4 & & & & $.037^{* * *}$ \\
$\begin{array}{l}\text { Neuroticism } \\
\text { Step 5 }\end{array}$ & -6.04 & 1.17 & $-.25^{* * *}$ & \\
Conscientiousness & 6.68 & 1.34 & $.22^{* * * *}$ & $.033^{* * *}$ \\
\hline
\end{tabular}

${ }^{*} p<.05, * * p<.01, * * * p<.001$

a Dummy coding was used to represent the five levels of race: Caucasian, African American, Hispanic/Latino, Asian, other 


\section{CHAPTER 4: DISCUSSION}

As predicted, personality traits and TEI were associated with complaints of sleep disturbance. TEI was the most robust predictor of both insomnia severity and nonrestorative sleep. Participants with higher TEI scores, indicating behavioral dispositions or self-perceived abilities related to better emotion regulation, perceived their sleep quality to be more restorative and had less severe insomnia problems. Results may indicate that individuals with higher TEI have more restorative sleep and less severe insomnia due to abilities to understand changes in mood and that daytime impairment may be related to their sleep or to other personal or environmental factors. These individuals may be more attuned to their emotionality and better at self-regulating emotional and physical distress.

Neuroticism was the second strongest personality variable predicting insomnia severity and nonrestorative sleep. Higher neuroticism was associated with poorer sleep overall, including increased insomnia severity and worse nonrestorative sleep quality. Those with higher neuroticism may perceive their sleep as being nonrestorative and additionally have more severe problems with insomnia due to excessive worry towards falling asleep, staying asleep, or being able to function adequately with a short sleep duration. These individuals may have heightened rumination and physical arousal at bed time, making it more difficult to fall asleep and leaving them vulnerable to difficulty sleeping. Additionally, these individuals may be more likely to have increased anxiety related to the amount of time spent awake and the effect this may have on their functioning the next day. 
Participants higher in traits of conscientiousness perceived their sleep quality as being more restorative and had less severe problems related to insomnia. Individuals with higher contentiousness have a tendency to act with self-discipline and commonly have planned rather than spontaneous behavior (Costa \& McCrae, 1985; 1992). Those with similar traits may have better sleep hygiene overall. Good sleep hygiene practices, a common feature of cognitive behavioral therapy for insomnia, may promote daytime alertness and better sleep (Morin, Culbert, \& Schwartz, 1994; Morin, et al., 1999; Morin, et al. 2006; Stepanski \& Wyatt, 2003). Sleep hygiene may additionally act as a protective factor related to the development of sleep problems and sleep disorders (Bartel, Gradisar, \& Williamson, 2015; Kline et al., 2014). These individuals may be more likely to set and adhere to routine sleep schedules which have been found to play a unique role in predicting insomnia severity among college students (Gellis, Stotsky, \& Taylor, 2014).

Although studies have not reported on associations between openness to experience and sleep disturbance, we predicted openness to experience would be positively associated with sleep. This predication was determined because openness to experiences is often identified as a positive trait and is partially characterized by attentiveness to feelings and new ideas, flexibility of thought, and intellectual curiosity (Costa et al., 1985; 1992). Inconsistent with predictions, results indicated participants higher in openness to experience perceived more severe problems with insomnia. Those who have higher openness to experience may have poor sleep hygiene due to a desire to upkeep social events and may engage in stimulating or spontaneous activities that disrupt sleep schedules.

Personality traits, as part of a group of factors, may be involved in precipitating and perpetuating the development of insomnia (Harvey, et al., 2014; van de Laar, et al., 2010). Sleep 
deprivation is negatively associated with emotional intelligence and is linked to impairments in interpersonal functioning, stress management, and coping (Killgore, et al., 2008). Additionally, insomnia and emotional dysregulation individually appear to be uniquely associated with psychopathology severity (Fairholme, Nosen, Nillni, Schumacher, Tull \& Coffey, 2013). There is concordance between results of this study and past research. In general, neuroticism is negatively, and conscientiousness is positively related to sleep (Harvey, et al., 2014; van de Laar, et al., 2010; Williams \& Moroz, 2009).

Findings of this study may aid in the understanding and improvement of assessment, diagnosis, and treatment of insomnia disorder and NRS. Adding a personality trait or TEI screening to clinical interviews could assist in the determination of whether additional components of treatment are warranted to address any individual differences which may negatively impact treatment outcomes. Personality traits and one's trait emotional intelligence may comprise predispositions to insomnia symptoms (Harvey, et al., 2014; van de Laar, et al., 2010) and perhaps complaints of NRS. Increases of neuroticism, decreases of conscientiousness, or decreases in TEI may play a role in development and maintenance of insomnia and daytime impairment of NRS (Killgore, et al., 2008; Ohayon, 2005; Williams \& Moroz, 2009). Furthermore, increases in conscientiousness and higher TEI could demonstrate a protective role to experiencing negative effects of poor sleep.

There are several limitations of the current study that warrant discussion. One constraint is that data from this study were gathered from participants at a single time point. Therefore, no causal conclusions can be made. Another limitation is that of generalizability. This study was conducted with undergraduate college students who make up a small percentage of the general population. To render this, using a system which reaches a variety of individuals (e.g. MTurk) 
could yield results with greater generalizability. Additionally, this research may be more appropriately directed to a population seeking treatment for insomnia or complaints of NRS.

Clinically based research studies may be better apt to answer questions related to characteristics of those who do or do not have successful outcomes and to identify possible modifications to current practice.

Future studies that address personality factors as potential moderators of the efficacy of insomnia treatments may be justified. Due to a high correlation of sleep problems with mood disorders, individuals high on neuroticism experiencing more sleep problems might be more resistant to successful therapy outcomes impacted by an increased worry at bedtime related to getting adequate sleep (Danielsson, Jansson-Fromark, Linton, Jutengren, \& Stattin, 2010; Ohayon, 2005; Tkachenko, Olson, Weber, Preer, Gogel, \& Killgore, 2014). Perhaps these individuals would benefit from treatment focused on maladaptive beliefs towards sleep and to specifically address concerns and anxiety related to sleep difficulties. Individuals high on neuroticism may also have poorer sleep hygiene and sleep habits than do those higher on conscientiousness or TEI. Highly conscientiousness or TEI may also be better at implementing components of treatment. Finally, since limited information is available, research focused on impairment for those with high levels of openness to experience may be warranted. 


\section{REFERENCES}

American Academy of Sleep Medicine. (2014). International classification of sleep disorders, ( $3^{\text {rd }}$ ed.). Westchester, IL. American Academy of Sleep Medicine.

American Psychiatric Association. (2013). Diagnostic and statistical manual of mental disorders: DSM-5, (5 ${ }^{\text {th }}$ ed.). Washington, D.C: American Psychiatric Publishing.

Barrash, J., Tranel, D., \& Anderson, S. W. (2000). Acquired personality disturbances associated with bilateral damage to the ventromedial prefrontal region. Developmental Neuropsychology, 18, 355-381.

Bartel, K. A., Gradisar, M., \& Williamson, P. (2015). Protective and risk factors for adolescent sleep: A meta-analytic review. Sleep Medicine Reviews, 21, 72-85.

Bastien, C. H., Vallières, A., \& Morin, C. M. (2001). Validation of the Insomnia Severity Index as an outcome measure for insomnia research. Sleep Medicine, 2, 297-307.

doi:10.1016/S1389-9457(00)00065-4

Benet-Martínez, V., \& John, O. P. (1998). Los Cinco Grandes across cultures and ethnic groups: Multitrait-multimethod analyses of the Big Five in Spanish and English. Journal of Personality and Social Psychology, 75, 729-750. doi:10.1037/0022-3514.75.3.729

Bogg, T., \& Roberts, B. W. (2004). Conscientiousness and health-related behaviors: A meta analysis of the leading behavioral contributors to mortality. Psychological Bulletin, 130, 887-919. doi:10.1037/0033-2909.130.6.887

Chiu, H. Y., Wang, M. Y., Chang, C. K., Chen, C. M., Chou, K. R., Tsai, J. C., \& Tsai, P. S. (2014). Early morning awakening and nonrestorative sleep are associated with increased minor non-fatal accidents during work and leisure time. Accident Analysis \& Prevention, 71, 10-14. doi:10.1016/j.aap.2014.05.002

Costa, P. T. Jr., \& McCrae, R. R. (1985). The NEO Personality Inventory. Odessa, FL: Psychological Assessment Resources.

Costa, P. T. Jr., \& McCrae, R. R. (1992). NEO PI-R Professional Manual. Odessa, FL: Psychological Assessment Resources, Inc.

Cooper, A., \& Petrides, K. V. (2010). A psychometric analysis of the Trait Emotional Intelligence Questionnaire-Short Form (TEIQue-SF) using item response theory. Journal of Personality Assessment, 92, 449-457. 
Danielsson, N. S., Jansson-Fromark, M., Linton, S. J., Jutengren, G., \& Stattin, H. (2010). Neuroticism and sleep-onset: What is the long-term connection? Personality and Individual Differences, 48, 463-468.

Davey, G. (2006). Encyclopaedic dictionary of psychology. London, England: Routledge.

Dawda, D., \& Hart, S. D. (2000). Assessing emotional intelligence: Reliability and validity of the Bar-On Emotional Quotient Inventory (EQ-i) in university students. Personality and Individual Differences, 28, 797-812.

Dorsey, C. M., \& Bootzin, R. R. (1997). Subjective and psychophysiologic insomnia: An examination of sleep tendency and personality. Biological Psychiatry, 41, 209-216. doi:10.1016/0006-3223(95)00659-1

Drake, C. L., Hays, R. D., Morlock, R., Wang, F., Shikiar, R., Frank, L., ... Roth, T. (2014). Development and evaluation of a measure to assess restorative sleep. Journal of Clinical Sleep Medicine, 10, 733-741, 741A-741E. doi:10.5664/jcsm.3860

Fairholme, C. P., Nosen, E. L., Nillni, Y. I., Schumacher, J. A., Tull, M. T., \& Coffey, S. F. (2013). Sleep disturbance and emotion dysregulation as transdiagnostic processes in a comorbid sample. Behaviour Research and Therapy, 51, 540-546.

Gellis, L. A., Stotsky, M. T., \& Taylor, D. J. (2014). Associations between sleep hygiene and insomnia severity in college students: Cross-sectional and prospective analysis. Behavior Therapy, 45, 806-816.

Goleman, D. (1995). Emotional intelligence. New York: Bantam Books.

Harvey, C. J., Gehrman, P., \& Espie, C. A. (2014). Who is predisposed to insomnia: A review of familial aggregation, stress-reactivity, personality and coping style. Sleep Medicine Reviews, 18, 237-247. doi:10.1016/j.smrv.2013.11.004

John, O. P, Donahue, E. M, \& Kentle, R. L. (1991). The Big Five Inventory--Versions 4a and 54. Berkeley, CA: University of California, Berkeley, Institute of Personality and Social Research.

John, O. P, Naumann, L. P, \& Soto, C. J. (2008). Paradigm shift to the integrative Big Five trait taxonomy: History, measurement, and conceptual issues. In O. P. John, R. W. Robins, \& L. A. Pervin (Eds.), Handbook of personality: Theory and research (pp. 114-158). New York, NY: Guilford Press.

Kahn, M., Sheppes, G., \& Sadeh, A. (2013). Sleep and emotions: bidirectional links and underlying mechanisms. International Journal of Psychophysiology, 89, 218-228. doi:10.1016/j.ijpsycho.2013.05.010 
Killgore, W. D. S., Kahn-Greene, E. T., Lipizzi, E. L., Newman, R. A., Kamimori, G. H., \& Balkin, T. J. (2008). Sleep deprivation reduces perceived emotional intelligence and constructive thinking skills. Sleep Medicine, 9, 517-526. doi:10.1016/j.sleep.2007.07.003

Kline, C. E., Irish, L. A., Buysse, D. J., Kravitz, H. M., Okun, M. L., Owens, J. F., \& Hall, M. H. (2014). Sleep hygiene behaviors among midlife women with insomnia or sleepdisordered breathing: The SWAN sleep study. Journal of Women's Health, 23, 894-903.

Lichstein, K. L., Durrence, H. H., Riedel, B. W., Taylor, D. J., \& Bush, A. J. (2004). Epidemiology of sleep: Age, gender, and ethnicity. Mahwah, NJ: Erlbaum.

Martinez-Pons, M. (1997). The relation of emotional intelligence with selected areas of personal functioning. Imagination, Cognition and Personality, 17, 3-13. doi: 10.2190/68VDDFXB-K5AW-PQAY

Morin, C. M. (1993). Insomnia: Psychological assessment and management. New York: Guilford Press.

Morin, C. M., Belleville, G., Bélanger, L., \& Ivers, H. (2011). The Insomnia Severity Index: Psychometric indicators to detect insomnia cases and evaluate treatment response. Sleep, $34,601-608$.

Morin, C. M., Bootzin, R. R., Buysse, D. J., Edinger, J. D., Espie, C. A., \& Lichstein, K. L. (2006). Psychological and behavioral treatment of insomnia: Update of the recent evidence (1998-2004). Sleep, 29, 1398-1414.

Morin, C. M., Culbert, J. P., Schwartz, S. M. (1994). Nonpharmacological interventions for insomnia: A meta-analysis of treatment efficacy. American Journal of Psychiatry, 151, 1172-1180.

Morin, C. M., Hauri, P. J., Espie, C. A., Spielman, A. J., Buysse, D. J., \& Bootzin, R. R. (1999). Nonpharmacologic treatment of chronic insomnia. An American Academy of Sleep Medicine review. Sleep, 22, 1134-1156.

Ohayon, M. M. (2005). Prevalence and correlates of nonrestorative sleep complaints. Archives of Internal Medicine, 165, 35-41. doi:10.1001/archinte.165.1.35

Ohayon, M. M., \& Roth, T. (2001). What are the contributing factors for insomnia in the general population? Journal of Psychosomatic Research, 51, 745-755. doi:10.1016/S00223999(01)00285-9

Petrides, K. V. (2009). Psychometric properties of the Trait Emotional Intelligence Questionnaire. In C. Stough, D. H. Saklofske, and J. D. Parker, Advances in the Assessment of Emotional Intelligence. New York: Springer (pp. 85-101). doi: 10.1007/978-0-387-88370-0_5 
Petrides, K. V., \& Furnham, A. (2001). Trait emotional intelligence: Psychometric Investigation with reference to established trait taxonomies. European Journal of Personality, 15, 425488. doi:10.1002/per.41

Petrides, K. V., \& Furnham, A. (2006). The role of Trait Emotional Intelligence in a genderspecific model of organizational variables. Journal of Applied Social Psychology, 36, 552-569. doi:10.1111/j.0021-9029.2006.00019.x

Rammstedt, B., \& John, O. P. (2007). Measuring personality in one minute or less: A 10-item short version of the Big Five Inventory in English and German. Journal of Research in Personality, 41, 203-212. doi:10.1016/j.jrp.2006.02.001

Roth, T., Jaeger, S., Jin, R., Kalsekar, A., Stang, P. E., \& Kessler, R. C. (2006). Sleep problems, comorbid mental disorders, and role functioning in the National Comorbidity Survey Replication. Biological Psychiatry, 60, 1364-1371. doi:10.1016/j.biopsych.2006.05.039

Roth, T., Zammit, G., Lankford, A., Mayleben, D., Stern, T., Pitman, V., ... Werth, J. L. (2010). Nonrestorative sleep as a distinct component of insomnia. Sleep, 33, 449-458.

Salovey, P., \& Mayer, J. (1990). Emotional intelligence. Imagination, Cognition and Personality, 9, 185-211.

Schutte, N. S., Malouff, J. M., Thorsteinsson, E. B., Bhullar, N., \& Rooke, S. E. (2007). A meta analysis of the relationship between emotional intelligence and health. Personality and Individual Differences, 42, 921-933. doi: 10.1016/j.paid.2006.09.003

Soto, C. J., John, O. P., Gosling, S. D., \& Potter, J. (2008). The developmental psychometrics of big five self-reports: Acquiescence, factor structure, coherence, and differentiation from ages 10 to 20. Journal of Personality and Social Psychology, 94, 718-737. doi:10.1037/0022-3514.94.4.718

Spinella, M. (2005). Prefrontal substrates of empathy: Psychometric evidence in a community sample. Biological Psychology, 70, 175-181. doi:10.1016/j.biopsycho.2004.01.005

Stepanski, E. J., \& Wyatt, J. K. (2003). Use of sleep hygiene in the treatment of insomnia. Sleep Medicine Reviews, 7, 215-225.

Stone, K. C., Taylor, D. J., McCrae, C. S., Kalsekar, A., \& Lichstein, K. L. (2008). Nonrestorative sleep. Sleep Medicine Reviews, 12, 275-288.

doi:10.1016/j.smrv.2007.12.002

Thorndike F. P., Ritterband, L. M., Saylor D. K., Magee J. C., Gonder-Frederick L. A., \& Morin C. M. (2011). Validation of the insomnia severity index as a web-based measure. Behavioral Sleep Medicine, 9, 216-223. 
Tkachenko, O., Olson, E. A., Weber, M., Preer, L. A., Gogel, H., \& Killgore, W. D. S. (2014). Sleep difficulties are associated with increased symptoms of psychopathology. Experimental Brain Research, 232, 1567-1574.

van de Laar, M., Verbeek, I., Pevernagie, D., Aldenkamp, A., \& Overeem, S. (2010). The role of personality traits in insomnia. Sleep Medicine Reviews, 14, 61-68. doi:10.1016/j.smrv.2009.07.007

Vernon, M. K., Dugar, A., Revicki, D., Treglia, M., \& Buysse, D. (2010). Measurement of nonrestorative sleep in insomnia: A review of the literature. Sleep Medicine Reviews, 14, 205-212. doi:10.1016/j.smrv.2009.10.002

Wilkinson, K., \& Shapiro, C. (2012). Nonrestorative sleep: symptom or unique diagnostic entity? Sleep Medicine, 13, 561-569. doi:10.1016/j.sleep.2012.02.002

Williams, P. G., \& Moroz, T. L. (2009). Personality vulnerability to stress-related sleep disruption: Pathways to adverse mental and physical health outcomes. Personality and Individual Differences, 46, 598-603. doi:10.1016/j.paid.2008.12.017 


\section{APPENDIX A: IRB CERTIFICATE}

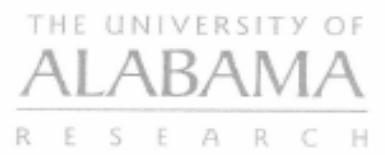

August 28, 2015

Sarah E. Emert, B.S.

Department of Psychology

College of Arts \& Sciences

The University of Alabama

Box 870348

Re: IRB \# 15-OR-192 (Revision \# 3) "Sleep, Personality, and Emotion"

Dear Ms. Emert:

The University of Alabama Institutional Review Board has reviewed the revision to your previously approved expedited protocol. The board has approved the change in your protocol.

Please remember that your approval period expires one year from the date of your original approval, June 12, 2015, not the date of this revision approval.

Should you need to submit any further correspondence regarding this proposal, please include the assigned IRB application number. Changes in this study cannot be initiated without IRB approval, except when necessary to eliminate apparent immediate hazards to participants.

Good luck with your research.

Sincerely,

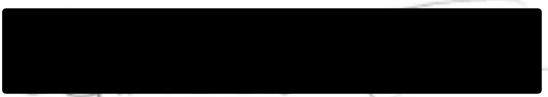

Carpantato T. Myles, MSM, CIM, CIP

Director \& Research Compliance Officer Office for Research Compliance 


\section{UNIVERSITY OF ALABAMA}

\section{INSTITUTIONAL REVIEW BOARD FOR THE PROTECTION OF HUMAN SUB.JECTS} REQUEST FOR APPROVAL OF RESEARCH INVOLVING HUMAN SUBJECTS

1. Identifying information

\begin{tabular}{|c|c|c|c|}
\hline & Principal Investigator & Second Investigator & Third Investigator \\
\hline Names: & Sarah E. Emert. B.S. & $\begin{array}{l}\text { Kenneth L. Lichstein. } \\
\text { Ph.D. }\end{array}$ & \\
\hline Department: & Psychology & Psychology & \\
\hline College: & Arts and Sciences & Arts and Sciences & \\
\hline University: & Univeristy of Alabama & Univeristy of Alabama & \\
\hline Address: & 505 Hackberry Lane & Box 870348 & \\
\hline & Tuscaloosa. AL. $35487-0348$ & $\begin{array}{l}505 \text { Hackberry Lane } \\
\text { Tuscaloosa, AL } 35487\end{array}$ & \\
\hline Telephone: & (520) $490-2510$ & (205) $348-4963$ & \\
\hline FAX: & $\mathrm{n} / \mathrm{a}$ & (205) 348-8648 & \\
\hline E-mail: & semertácrimson.ua.edu & lichstein/aua.edu & \\
\hline
\end{tabular}

Title of Research Project: Sleep. Personality, and Emotion

Date Submitted: $8 / 24 / 2015$

Funding Source: N/A

Type of Proposal $\square$ New

UA faculty or staff member signature:

11. NOTIFICATION OF IRB ACTION(to be completed by IRB):

Type of Review: Full board Expedited

\section{IRB Action:}

\section{Rejected}

Tabled Pending Revisions

Approved Pcnding Revisions

$\checkmark$ subjects.

Approval is effective until the following date: $6-1 /-16$.

Items approved: Research protocol

__ Informed consent

Recruitment materials

Date:

Date:

Date:

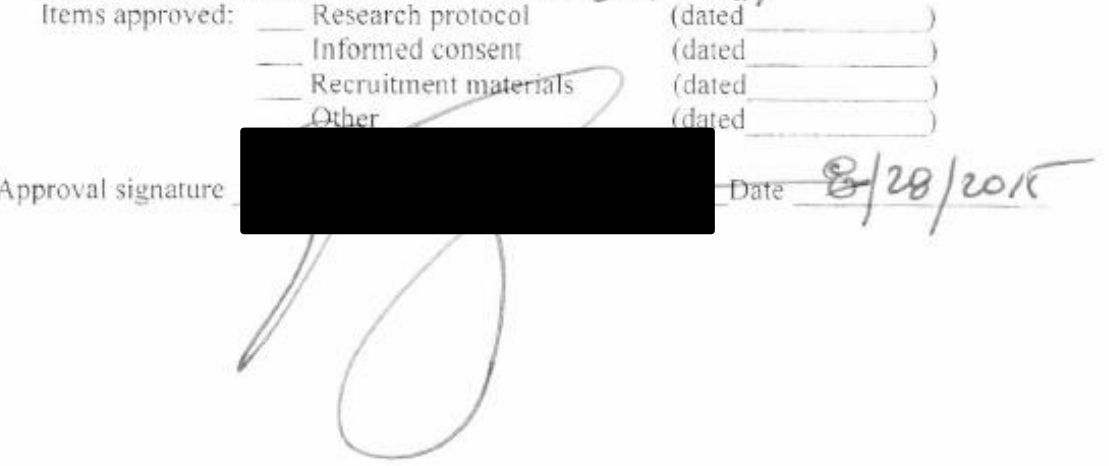

